\title{
Indications for flexible fiberoptic bronchoscopy and its safety in the very elderly
}

\author{
R. D'Ippolito1, A. Foresi², C. Castagnetti1, S. Gesualdi1, \\ A. Castagnaro', E. Marangio ${ }^{1}$, D. Olivieri ${ }^{1}$
}

ABSTRACT: Indications for flexible fiberoptic bronchoscopy and its safety in the very elderly. R. D'Ippolito, A. Foresi, C. Castagnetti, S. Gesualdi, A. Castagnaro, E. Marangio, D. Olivieri.

Aim. To evaluate the indications and the safety of fiberoptic bronchoscopy (FOB) with bronchoalveolar lavage (BAL), protected specimen brushing (PSB), endobronchial biopsy (EBB), and transbronchial biopsy (TBB) in a population of very elderly patients.

Methods. We performed a retrospective study of all adult patients, aged 50 years or older, who underwent FOB in the Bronchology Unit of the University of Parma Hospital between 1 January, 2003 and 31 April, 2005. Bronchoscopy records of $\mathbf{4 3 6}$ consecutive patients, including 191 patients, 75 yrs of age and older ("very elderly"; $=>75 \mathrm{yrs}$ ), were reviewed.
Results. Patients aged $<75$ years and aged $=/>75$ years were no different with regard to gender, $\mathrm{BMI}$, baseline $\mathrm{FEV}_{1} / \mathrm{FVC}$ ratio, baseline $\mathrm{SaO}_{2}$, and blood pressure. The primary indication in patients aged $<75$ years, was to assist in the diagnosis of a pulmonary mass of unknown aetiology $(33 \%)$ and to remove secretions in the very elderly patients $(31 \%)$. Indications for FOB and sampling procedures in the two groups were similar. Approximately $30 \%$ of patients in each group required supplemental oxygen during the procedure and fever occurred in $9.2 \%$ and $10.3 \%$ of patients, respectively.Hypertension and bleeding were relatively rare and did not occur more often in the very elderly.

Conclusions. Indication for FOB did not vary with age and adverse events in both groups were uncommon and generally not severe.

Monaldi Arch Chest Dis 2007; 67: 1, 23-29.

Keywords: Bronchoscopy, age, elderly, safety.

1 Department of Clinical Sciences, Section of Respiratory Diseases, University of Parma, Parma

2 Respiratory Pathophysiology Unit, Sesto San Giovanni Hospital, Sesto San Giovanni, Italy.

Correspondence: Raffaele D’Ippolito; Clinica Pneumologica, Padiglione "G. Rasori”, Università di Parma, Via Rasori 10, 43100 Parma, Italy; e-mail: rdippolito@ao.pr.it

\section{Introduction}

Flexible fiberoptic bronchoscopy (FOB) and associated procedures have a relevant diagnostic and therapeutic value $[1,2]$. In general, complication rates are low with a morbidity of $<1 \%$ and $0 \%$ mortality [3, 4]. FOB is now commonly performed, and the number of elderly patients undergoing the procedure is increasing [5]. Guidelines on the diagnostic use of FOB by the British Thoracic Society do not consider age to be a barrier to its application [1]. This statement is mainly based on studies performed in the 1980's [6-9]. However, only a minority of patients included in these studies were aged 75 years and older (very elderly). More recently, a group of 219 people, aged 70 years or older, who underwent FOB procedures, were prospectively examined [5]. Risk for adverse events was found to increase with age [5]. In addition indications for FOB also changed with the patients' age [5-10].

The number of applications for FOB procedures in elderly patients will likely grow tremen- dously in the near future with expanding applications in clinical medicine [11] and research [12-14]. In particular, the role of FOB procedures in lung cancer continues to expand. Since the peak incidence of lung cancer is now between 75 and 80 years of age [15, 16], FOB is likely to be performed more frequently in very elderly patients.

The purpose of this study was to retrospectively examine the indications and the frequency of adverse events of FOB and of its associated routine procedures, in very elderly patients attending a teaching university hospital.

\section{Methods}

FOB data was obtained by comprehensive review of inpatient and outpatient charts and bronchoscopy reports from the database of the Bronchoscopy Unit. We retrospectively examined the records of all patients, aged $>50$ years, who underwent FOB between 1 January, 2003 and 31 April, 
2005, at the University of Parma Hospital for clinical purposes. FOB reports, completed by the attending bronchoscopist and technician, contained indications, anaesthesia, procedures, findings, and adverse events. Adverse events were monitored for 24 hours following a standard protocol for filling out bronchocopy reports.

\section{Bronchoscopy procedures}

FOB and diagnostic techniques were performed as recommended by the American Thoracic Society [17], the British Thoracic Society [1], and ACCP [18], slightly modified.

After obtaining informed consent, patients fasted for at least 8 hours prior to the procedure. A peripheral intravenous catheter remained inserted. All patients were in the supine position and underwent bronchoscopy by a flexible fiberoptic bronchoscope (Olympus PE; Japan). All subjects received IM atropine $(0.5 \mathrm{mg})$, unless contraindicated. If necessary, IM diazepam was administered for sedation $(5-15 \mathrm{mg})$. Local anaesthesia was performed by inhalation of an aerosol solution of $2 \mathrm{ml}$ of $2 \%$ lidocaine followed by the sucking of a $20 \mathrm{mg}$ tablet of tetracaine $15 \mathrm{~min}$ before FOB. The bronchoscope was inserted nasally and the oral route was used as a second choice. Three aliquots of lidocaine $(100 \mathrm{mg}$ in $5 \mathrm{ml})$ were administered during FOB (glottis, left and right bronchus level). Sampling during the procedure was individualised for each case and included bronchoalveolar lavage (BAL), protected specimen brushing (PSB), endobronchial biopsy (EBB), and transbronchial biopsy (TBB). The choice of sampling procedure during FOB was at the bronchoscopist's discretion. The standard sequence of sampling techniques used was: BAL, PSB, EBB, followed by TBB. During all bronchoscopy procedures, patients were connected to a computerised electrocardiographic recorder and pulse oxymeter (Cardiolife Nihon Kohden Defibrillator, model TEC-7521G; Tokio; Japan), using a finger probe on the left hand. Blood pressure was measured through a sphygmomanometer. All bronchospy procedures were done under the supervision of an expert operator (RD), assisted by a trained bronchoscopy technician. All patients signed an informed consent before FOB.

EBB were taken from various sites using a coated disposable biopsy forceps (Olympus FB 19c-1 or FB 211; Tokio; Japan). A minimum of three biopsies were taken.

PSB was done where required using a disposable cytology brush (Olympus BC 202d-3010; Tokio; Japan) [19].

BAL was carried out as previously described [20]. The bronchoscope was wedged into a segment of the right middle lobe, and three $50-\mathrm{mL}$ aliquots of sterile saline solution, warmed to $37^{\circ} \mathrm{C}$, were instilled into the subsegmental bronchus. Fluid was gently aspirated immediately after each aliquot was introduced, and collected in a sterile container.
TBB was performed under fluoroscopic guidance. The bronchoscope was wedged in the desired segment. The forceps (Olympus FB 19c-1 or FB 211; Tokio; Japan) were then extended to the lung periphery to reach the desired position. Patients were asked to exhale and the open forceps was advanced and then closed. The procedure was repeated 3 to 5 times to obtain adequate samples.

Exclusion criteria for FOB which included PSB, EEB and TBB were: platelet count $<50,000$ thrombocytes $\cdot \mathrm{mm}^{-3}$; activated partial thromboplastin time $\geq 50$ s or prothrombin time $\leq 75 \%$ ); and haemodynamic instability. Had a severe adverse event occurred, trained technicians and an anaesthetist would have been available with resuscitation equipment and standard protocols. Once the procedure was completed, outpatients were taken to a recovery area for about 180 minutes before being discharged.

\section{Laboratory Data}

Laboratory data from each patient was recorded and included WBC count, urea, prothrombin time, and platelet count.

Flow-volume loops and arterial blood gasses were also measured when warranted, based on the patients' characteristics and their clinical conditions.

\section{Adverse events}

All adverse events due to FOB and within 24 hours following the procedure, were detected by a retrospective chart review [21]. Adverse events were defined as events of FOB and related procedures occurring during or after $\mathrm{FOB}$, even if there were no apparent causal relationship to FOB.

Prospectively recorded adverse events included the following: haemoglobin oxygen desaturation (below 90\%) requiring supplemented oxygen or an increase in the fraction of inspired oxygen delivered by nasal cannula; hypotension/hypertension; bleeding, described as mild or severe by the bronchoscopist; arrhythmia requiring discontinuation of the procedure; bronchospasm; stridor/tirage; fever (defined as an axillary body temperature $\geq 38^{\circ} \mathrm{C}$ ); vomiting; myocardial infarction; pneumothorax; life-threatening cardiac arrhythmia; or death. An event was considered major if it resulted in interruption of the procedure. Adverse events were documented either at the end of FOB or at clinic review. Chest radiographs were obtained to determine whether a pneumothorax had occurred after FOB with TBB.

\section{Statistical analysis}

Data was presented as mean \pm SD. Differences between groups (age $<75$ years or $=>75$ years) were examined by unpaired Student's $t$ test, by 


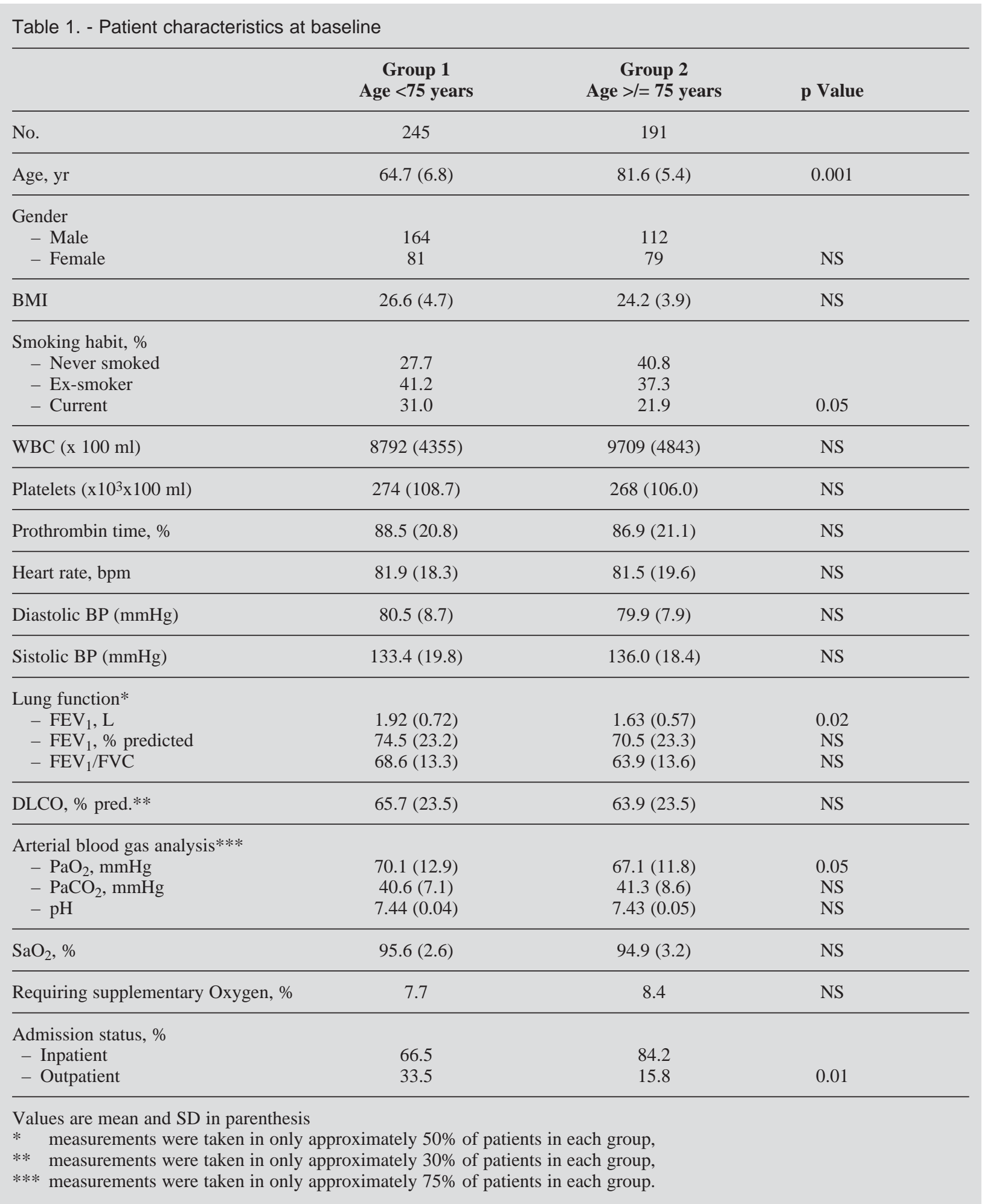

Fisher's Exact Test, and by $\chi^{2}$ for trends. A $p$ value of 0.05 was considered to indicate statistical significance.

\section{Results}

We selected bronchoscopy records of 301 patients who underwent FOB. FOB was performed transnasally in $98 \%$ of cases. To facilitate the in- terpretation of the results, patients were divided into two groups according to their age (group 1: age $<75$ years, $n=245$ and group 2 : age $=>75$ years, $\mathrm{n}=191)$. Their baseline characteristics are shown in table 1. Mean age was 64.7 yrs in group 1 and was 81.6 yrs in group 2. Group 2 included significantly more patients who had never smoked $(p<0.05$; table 1$)$, and more inpatients ( $<<0.01$; table 1$)$. Anthropometric characteristics and clinical data were well balanced in the two groups. As expected, 
Table 2. - Indications and sampling methods

\begin{tabular}{|c|c|c|c|}
\hline & $\begin{array}{c}\text { Group } 1 \\
\text { Age }<75 \text { years } \\
\mathrm{N}=\mathbf{2 4 5}\end{array}$ & $\begin{array}{c}\text { Group } 2 \\
\text { Age }>/=75 \text { years } \\
N=191\end{array}$ & p Value \\
\hline \multicolumn{4}{|l|}{ Indications, \% } \\
\hline - Mass & 33.9 & 21.9 & \\
\hline - Hemoptysis & 7.3 & 4.3 & \\
\hline - Collapse or consolidation & 19.2 & 24.6 & \\
\hline - Retained secretions & 18.8 & 31.9 & \\
\hline - Others & 20.8 & 17.3 & NS \\
\hline \multicolumn{4}{|l|}{ Sampling method, \% } \\
\hline$-\mathrm{BAL}$ & 21.1 & 16.5 & \\
\hline - PSB & 21.6 & 26.8 & \\
\hline$-\mathrm{EBB}$ & 42.2 & 45.4 & \\
\hline$-\mathrm{TBB}$ & 15.2 & 11.3 & NS \\
\hline
\end{tabular}

Table 3. - Bronchoscopy adverse events

\begin{tabular}{|c|c|c|}
\hline & $\begin{array}{c}\text { Group } 1 \\
\text { Age }<75 \text { years } \\
\text { N=245 }\end{array}$ & $\begin{array}{c}\text { Group } 2 \\
\text { Age }>/=75 \text { years } \\
N=191\end{array}$ \\
\hline Supplemental Oxygen* & $86(35.1 \%)$ & $66(34.6 \%)$ \\
\hline Hypertension & $9(3.7 \%)$ & $7(3.7 \%)$ \\
\hline Hypotension & $1(0.4 \%)$ & 0 \\
\hline Bleeding/hemorrhage & $8(3.3 \%)$ & $4(2.1 \%)$ \\
\hline Bronchospasm & $2(0.8 \%)$ & $3(1.6 \%)$ \\
\hline Stridor/Tirage/Laryngospasm & $2(0.8 \%)$ & 0 \\
\hline Fever* & $22(9.4 \%)$ & $21(10.9 \%)$ \\
\hline Vomiting & $2(0.8 \%)$ & 0 \\
\hline Myocardial infarction & 0 & 0 \\
\hline Pneumothorax & $1(0.4 \%)$ & 0 \\
\hline Arrhythmia & 0 & 0 \\
\hline Death & 0 & 0 \\
\hline
\end{tabular}

mean $\mathrm{PaO}_{2}$ value was lower in the very elderly $(p<0.05$, table 1$)$. Long-term oxygen supply was administered to $7.7 \%$ and $8.4 \%$ of patients, respectively (table 1).

The primary clinical indications for FOB and the sampling methods used are reported in table 2 . The primary indication in patients aged $<75$ years, was to assist in the diagnosis of a pulmonary mass of unknown aetiology (33\%) and to remove secretions in the very elderly patients $(31 \%)$. Differences between groups were not statistically significant. FOB without any additional procedure was more frequently performed in the very elderly (125 patients vs 100 patients; $p<0.01)$. Sampling methods in the two groups were not different (table 2). EBB was the most commonly performed procedure in both groups.

Bronchoscopy adverse events are listed in table 3. There was no death associated with FOB. None of patients required mechanical ventilation after FOB procedures. No procedure was interrupted as a result of a major adverse event. Patients requiring supplemental oxygen due to acute worsening of oxygenation status during or after FOB were similar in the two groups (approximately $35 \%$; table 3 ). Overall, patients requiring supple- 
mental oxygen did not have significantly lower baseline $\mathrm{FEV}_{1}$ values, expressed as percent of predicted $(72.7 \%$ vs $77.4 \%)$. Similar results were found within each patient subgroup. Fever developed in about $10 \%$ of patients in both groups (table 3 ). Baseline WBC count in those who developed fever, was not higher compared to those who did not. Local bleeding at the biopsy sites was generally minimal. Only two patients in group 1 required intervention with topical adrenaline. Pneumothorax occurred in only one patient who underwent FOB without any biopsy procedure.

\section{Discussion}

Our results suggest that indications for FOB did not vary remarkably with age. This confirms the conclusions of a comprehensive review article [10]. However a recent study suggests an increasing indication for mass and pulmonary nodules with increasing age [5]. This discrepancy is likely related to different prevalence of the diseases, differences in institutional missions, and to selection criteria for the patients. In addition, we arbitrarily established a predefined age cut-off of 75 years whereas others included all adults $(>=18$ years) [5]. When examining data in the same age range (i.e. >50 years) from this study [5], it seems that there is very little difference in bronchoscopy indication, as in our report. Since aging is a continuum, defying the age cut-off is always arbitrary. However, changes in population demography and pathophysiological condition suggest that the current cut-off of 65 years should be changed to those over 75 years [22].

Fever after FOB is a well-known adverse reaction. Its incidence in adults is variable according to different reports. Fever was associated with FOB in $2.5 \%$ of 281 procedures in the study by [23]. While in other studies 5\% [24] and 16\% [25] of adult patients presented a fever after FOB. We found an incidence of fever in approximately $10 \%$ of patients. Thus, our results are in line with previous reports. Differences in underlying pulmonary diseases, diagnostic techniques during $\mathrm{FOB}$, inclusion of immunocompromised patients, prior antibiotic or corticosteroid therapy and the definition of fever may account for the discrepancies in the incidence of fever. Various clinical parameters were associated with fever after FOB by univariate analysis. However, only the final diagnosis of pulmonary tuberculosis and the severity of bleeding were identified as independent risk factors for fever by multivariate analysis [24]. According to previous studies, postbronchoscopy fever is associated with advanced age [25-27]. Our results do not support these findings [24].

Although there is a debate concerning the need for sedation in FOB [28, 29], we always performed non-sedated bronchoscopy with very few exceptions. Rather old studies suggested that sedation was implicated as the cause of major averse events resulting from FOB $[3,30]$. More recent studies did not confirm these findings [31, 32]. Sedation, in combination with topical anaesthesia and mechanical obstruction from the endoscope, results in hypoxemia [29]. Although we did not routinely apply sedation, a consistent rate of patients (approximately 1/3) in both groups required supplemental oxygen. Our results are consistent with previous findings showing that $86 \%$ of patients did not require routine oxygen supplementation [31]. At odds with previous findings [31, 33], we found that $F E V_{1}$ is not a risk factor for oxygen supplementation even in the very elderly and a fall in oxygen saturation may occur at any $\mathrm{FEV}_{1}$ level. We would expect a higher rate in the very elderly since baseline $\mathrm{PaO}_{2}$ was lower at baseline in this group. The absence of difference likely reflects diversity in the bronchoscopy procedures between the two groups and could also be related to patient selection. It is unlikely that more severely ill elderly patients would undergo any FOB due to the higher risk of serious side effects and to comorbidities.

Serious cardiac adverse events, such as atrial and ventricular arrhythmias, myocardial ischemia, angina, and cardiac arrest are uncommon [3, 5, 30, 34-36]. They have been linked to sedation, hypoxemia [37], passage of the bronchoscope through the vocal cords [34], and pre-treatment with lidocaine anaesthetic. Thus, the absence of any serious cardiac event in our population could likely be explained by the absence of sedation, by accurate and quick oxygen supplementation during FOB, by the professional skill and experience of the bronchoscopist and by the amount of lidocaine administered to the patients. Although the cough reflex is reduced in the elderly [38], we did not change their premedication.

Approximately $300 \mathrm{mg}$ of lidocaine was administered. Despite the fact that this exceeded the recommended highest dose [1], it has been demonstrated not to be toxic $[30,40]$ even in the very elderly [41], despite a prolonged half-time elimination [42]. Moreover it might exert a protective effect against the development of major arrhythmias during the procedure [43].

Deaths are also mostly due to cardiovascular effects. No death occurred in our population of very elderly, indirectly suggesting that even in this population the death rate during FOB is rather low [36].

Some limitations of this study must be acknowledged:

First, FOB was carried out in a dedicated operational unit of the university, with the cooperation of skilled personnel.

Second, the absence of differences in serious side effects between the two age groups were indeed related to their low prevalence, but we cannot exclude that it might also be related to patient selection since it is unlikely that more severely ill elderly patients would undergo FOB due to the high risk and to comorbidities; and

Third, TBB was performed in relatively few patients to draw any firm conclusion on the safety of this procedure in the elderly. The overall 
message may not be applied to a large variety of medical settings. A prospective evaluation of risk factors for bronchoscopy procedures due to the associated comorbidities in the elderly is still lacking.

In conclusion, despite the wide use of FOB as a diagnostic and therapeutic tool, to our knowledge, this is the first study to examine the indication and safety profile in a large population of very elderly patients. This retrospective study conducted in a university hospital clearly shows that FOB is safe and adverse events are uncommon and generally not severe. Transient fever is a relatively frequent adverse event, whereas other minor or major adverse events are less frequent and not associated with the patients' age. These results in "octogenarians" should help quash any reluctance to consider FOB even in the very elderly.

Acknowledgements: We acknowledge the nursing and technical assistance provided by D'Auder MG, De Caprio MT, Di Maria MV, Facini OR, Salati MP.

\section{References}

1. British Thoracic Society guidelines on diagnostic flexible bronchoscopy. Thorax 2001; 56: i1-i21.

2. Beamis JF, Becker HD, Cavaliere S, et al. ERS/ATS statement on interventional pulmonology: Chairmen: C.T. Bolliger, P.N. Mathur. Eur Respir J 2002; 19: 356-373

3. Credle SW, Smiddy JF, Elliott RC. Complications of fiberoptic bronchoscopy. Am Rev Respir Dis 1974; 109, 67-72.

4. Pue CA, Pacht ER. Complications of fiberoptic bronchoscopy at a university hospital. Chest 1995; 107: 430-432.

5. Hehn BT, Haponik E, Rubin HR, et al. The relationship between age and process of care and patient tolerance of bronchoscopy. J Am Geriatr Soc 2003; 51: 917-922.

6. Brandstetter RD, Croce SA, Schiaffino E, et al. Flexible fiberoptic bronchoscopy in the elderly. $N Y$ State $J$ Med 1984; 84: 546-548

7. Knox AJ, Mascie-Taylor BH, Page RL. Fibreoptic bronchoscopy in the elderly: 4 years' experience. $B r J$ Dis Chest 1988; 82: 290-293.

8. Macfarlane JT, Storr A, Wart MJ, et al. Safety, usefulness and acceptability of fibreoptic bronchoscopy in the elderly. Age Ageing 1981; 10: 127-131.

9. O'Hickey S, Hilton AM. Fibreoptic bronchoscopy in the elderly. Age Ageing 1987; 16: 229-233.

10. Hehn B, Haponik EF. Flexible bronchoscopy in the elderly. Clin Chest Med 2001; 22: 301-309.

11. Hopkinson NS, Toma TP, Hansell DM, et al. Effect of bronchoscopic lung volume reduction on dynamic hyperinflation and exercise in emphysema. Am J Respir Crit Care Med 2005; 171: 453-460.

12. El Solh AA, Pietrantoni C, Bhat A, et al. Colonization of dental plaques: a reservoir of fespiratory pathogens for hospital-acquired pneumonia in institutionalized elders. Chest 2004; 126: 1575-1582.

13. Hattotuwa K, Gamble EA, O'Shaughnessy T, et al. Safety of bronchoscopy, biopsy, and BAL in research patients with COPD. Chest 2002; 122: 1909-1912.

14. Kikuchi E, Yamazaki K, Sukoh N, et al. Endobronchial ultrasonography with guide-sheath for peripheral pulmonary lesions. Eur Respir J 2004; 24: 533-537.
15. Booton R, Jones M, Thatcher N. Lung cancer: Management of lung cancer in elderly patients. Thorax 2003; 58: 711-720.

16. Brown JS, Eraut D, Trask C, Davison AG. Age and the treatment of lung cancer. Thorax 1996; 51: 564-568.

17. American Thoracic Society. Clinical role of bronchoalveolar lavage in adults with pulmonary disease. Am Rev Respir Dis 1990; 142: 481-486.

18. Ernst A, Silvestri GA, Johnstone D. Interventional pulmonary procedures: guidelines from the american college of chest physicians. Chest 2003; 123: 1693-1717.

19. Ahmad M, Livingston DR, Golish JA, et al. The safety of outpatient transbronchial biopsy. Chest 1986; 90: 403-405.

20. D'Ippolito R, Foresi A, Chetta A, et al. Induced sputum in patients with newly diagnosed sarcoidosis: comparison with bronchial wash and BAL. Chest 1999; 115: 1611-1615.

21. Dunagan DP, Burke HL, Aquino SL, et al. Fiberoptic bronchoscopy in coronary care unit patients: indications, safety, and clinical implications. Chest 1998; 114: $1660-1667$.

22. Horimo H. Reviewing the definition of elderly. Nippon Ronen Igakkai Zasshi. 2006; 43: 27-34.

23. Strumpf IJ, Feld MK, Cornelius MJ, et al. Safety of fiberoptic bronchoalveolar lavage in evaluation of interstitial lung disease. Chest 1981; 80: 268-271.

24. Um SW, Choi CM, Lee CT, et al. Prospective analysis of clinical characteristics and risk factors of postbronchoscopy fever. Chest 2004; 125: 945-952.

25. Pereira W, Jr., Kovnat DM, Khan LN, et al. Fever and pneumonia after flexible fiberoptic bronchoscopy. Am Rev Respir Dis 1975; 112: 59-64.

26. Krause A, Hohberg B, Heine F, et al. Cytokines derived from alveolar macrophages induce fever after bronchoscopy and bronchoalveolar lavage. Am J Respir Crit Care Med 1997; 155: 1793-1797.

27. Nelson ME, Wald TC, Bailey K, et al. Intrapulmonary cytokine accumulation following BAL and the role of endotoxin contamination. Chest 1999; 115 : 151-157.

28. Pickles J, Jeffrey M, Datta A, et al. Is preparation for bronchoscopy optimal? Eur Respir J 2003; 22: $203-$ 206.

29. Smyth CM, Stead RJ. Survey of flexible fibreoptic bronchoscopy in the United Kingdom. Eur Respir $J$ 2002; 19: 458-463.

30. Matot I, Kramer MR, Glantz L, et al. Myocardial ischemia in sedated patients undergoing fiberoptic bronchoscopy. Chest 1997; 112: 1454-1458.

31. Jones AM, O'Driscoll R. Do all patients require supplemental oxygen during flexible bronchoscopy? Chest 2000; 119: 1906-1909.

32. Putinati S, Ballerin L, Corbetta L, et al. Patient satisfaction with conscious sedation for bronchoscopy. Chest 1999; 115: 1437-1440.

33. Davies L, Mister R, Spence DP, et al. Cardiovascular consequences of fibreoptic bronchoscopy. Eur Respir J 1997; 10: 695-698.

34. Katz AS, Michelson EL, Stawicki J, et al. Cardiac arrhythmias. Frequency during fiberoptic bronchoscopy and correlation with hypoxemia. Arch Intern Med 1981; 141: 603-606.

35. Lindholm CE, Ollman B, Snyder JV, et al. Cardiorespiratory effects of flexible fiberoptic bronchoscopy in critically ill patients. Chest 1978; 74: 362-368

36. Suratt PM, Smiddy JF, Gruber B. Deaths and complications associated with fiberoptic bronchoscopy. Chest 1976; 69: 747-751.

37. Shrader DL, Lakshminarayan S. The effect of fiberoptic bronchoscopy on cardiac rhythm. Chest 1978; 73 : 821-824 
38. Newnham DM, Hamilton SJ. Sensitivity of the cough reflex in young and elderly subjects. Age Ageing 1997; 26: $185-188$

39. Langmack EL, Martin RJ, Pak J, et al. Serum lidocaine concentrations in asthmatics undergoing research bronchoscopy. Chest 2000; 117: 1055-1060.

40. Loukides S, Katsoulis K, Tsarpalis K, et al. Serum concentrations of lignocaine before, during and after fiberoptic bronchoscopy. Respiration 2000; 67: 13-17.

41. Watts MR, Geraghty R, Moore A, et al. Premedication for bronchoscopy in older patients: a double-blind comparison of two regimens. Respir Med 2005; 99: 220226.

42. Abernethy DR, Greenblatt DJ. Impairment of lidocaine clearance in elderly male subjects. J Cardiovasc Pharmacol 5, 1093-1096. 1983.

43. Elguindi AS, Harrison GN, Abdulla AM, et al. Cardiac rhythm disturbances during fiberoptic bronchoscopy: a prospective study. J Thorac Cardiovasc Surg 1979; 79: 557-561.

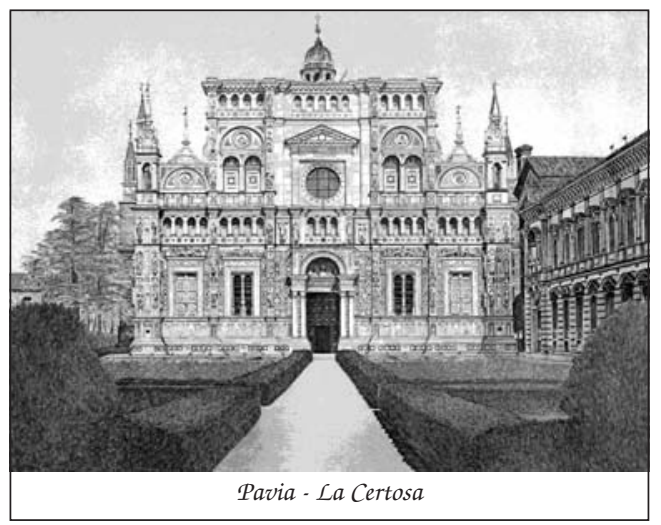

